\title{
Magnetic properties of the QCD medium
}

\author{
Claudio Bonati \\ Dipartimento di Fisica, Università di Pisa and INFN, Largo Pontecorvo 2, I-56127 Pisa, Italy \\ E-mail: bonati@df.unipi.it
}

\section{Massimo D'Elia}

Dipartimento di Fisica, Università di Pisa and INFN, Largo Pontecorvo 2, I-56127 Pisa, Italy

E-mail: deliaedf.unipi.it

\section{Marco Mariti*}

Dipartimento di Fisica, Universià di Pisa and INFN, Largo Pontecorvo 2, I-56127 Pisa, Italy

E-mail: mariti@df.unipi.it

\section{Michele Mesiti}

Dipartimento di Fisica, Universià di Pisa and INFN, Largo Pontecorvo 2, I-56127 Pisa, Italy

E-mail: michele.mesiti@pi.infn.it

\section{Francesco Negro}

Dipartimento di Fisica, Universià di Pisa and INFN, Largo Pontecorvo 2, I-56127 Pisa, Italy

E-mail: fnegro@pi.infn.it

\section{Francesco Sanfilippo}

School of Physics and Astronomy, University of Southampton, Southampton SO17 1BJ, United

\section{Kingdom}

E-mail: f.sanfilippoesoton.ac.uk

\begin{abstract}
We present our study of the QCD medium magnetic properties. We use $N_{f}=2+1$ quark flavors QCD at the physical point, with Symanzik tree level improved gauge action and stout improved staggered fermions. We find that the strongly interacting matter behaves as a paramagnet in the range of temperatures $(100-400) \mathrm{MeV}$, with a sharp increase of the magnetic susceptibiliy at the deconfinement crossover. For magnetic field values expected during off-central heavy ion collisions, we found that the magnetic contribution to the pressure can reach up to the $40 \%$ of the total pressure near the crossover transition. Finally we discuss preliminary results on higher order magnetic contributions to the free energy.
\end{abstract}

The 32nd International Symposium on Lattice Field Theory,

23-28 June, 2014

Columbia University New York, NY

\footnotetext{
* Speaker.
} 


\section{Introduction}

In the last years many efforts have been devoted to the study of strongly interacting systems in the presence of external magnetic fields with energy close to the QCD scale [1, 2, 3, 4]. From the phenomenological point of view one expects to find these conditions during off-central heavy ion collisions $\left(e B \simeq 0.1 \mathrm{GeV}^{2}\right)$ [5], in compact astrophysical objects $\left(e B \simeq 10^{-6} \mathrm{GeV}^{2}\right)[6]$ and in early cosmological stages $\left(e B \simeq 1 \mathrm{GeV}^{2}\right)$ [7]. A full understanding of the QCD properties in the presence of such fields is clearly of fundamental interest to describe these phenomena, furthermore it can help to clarify many unsolved aspects of the theory.

If we consider a thermal medium in the presence of external magnetic fields, a first issue we may address is the dependence of thermodynamical quantities on such background. In particular, a relevant observable is the free energy density of the system. To study the dynamics of strongly interacting systems beyond perturbation theory one can use the lattice formulation of QCD, where an external magnetic field can be easily added to the discretized theory inserting proper $U(1)$ phases in the Dirac operator [8]. However, due to the toroidal geometry of our lattice space-time (imposed to reduce finite size effects), we cannot arbitrarly choose the magnetic field values in our simulations, which instead get quantized. For a system with minimum charge $q=\frac{|e|}{3}$, one arrives to the following quantization condition [9]:

$$
e B=\frac{6 \pi b}{a^{2} L_{\mu} L_{v}}, \quad b \in \mathbb{Z}
$$

where $L_{\mu}, L_{v}$ are the lattice extents along the directions orthogonal to the magnetic field. This quantization condition hinders the possibility to directly compute on the lattice free energy derivatives with respect to the magnetic field. In the last years several methods have been proposed to circumvent this problem (see $[10,11,12,13,14])$. In this work we present our latest determination of the magnetic susceptibility of QCD matter and of the pressure dependence on $B$ [11]. Finally we discuss preliminary results of higher order terms in the free energy dependence on the magnetic field.

\section{The method}

We determine the free energy density dependence on the magnetic filed $B$ by measuring the finite free energy differences $[10,11]$ :

$$
\Delta f(b, T)=f(b, T)-f(b-1, T)=\int_{b-1}^{b} \frac{\partial f(\bar{b}, T)}{\partial \bar{b}} d \bar{b}
$$

where $b \in \mathbb{Z}$ is the magnetic quantum in Eq. 1.1. This can be done defining a trajectory in parameter space which interpolates from the system with a uniform magnetic background $(b-1)$ to the system with uniform field $b$. This procedure defines an analitic extension of the free energy $f(b, T)$ for unquantized values of the magnetic field, where $f$ does not refer anymore to the free energy density of a system with a uniform magnetic field. Indeed, for $b \notin \mathbb{Z}, f(b, T)$ is just an interpolating function between different magnetic quanta, which reduces to the free energy density of the physical system for $b \in \mathbb{Z}$. Among the possible different paths, our choise is to move straight from 
$(b-1)$ to $b$, introducing a real valued magnetic field $b \notin \mathbb{Z}$ which explicitly breaks the quantization condition. Finally we obtain $\Delta f(b, T)$ integrating the derivative $\frac{\partial f(b, T)}{\partial b}$ over the above defined path, where $\frac{\partial f(b, T)}{\partial b}$ does not correspond to the magnetization of the system, but it is just the derivative of the chosen interpolating funtion.

The integrand in Eq. 2.1 have been computed on the lattice by measuring the operator:

$$
\frac{\partial f}{\partial b}=\frac{1}{4 a^{4} L_{t} L_{s}^{3}} \sum_{f=u, d, s}\left\langle\operatorname{tr}\left\{\frac{\partial D^{f}}{\partial b} D^{f^{-1}}\right\}\right\rangle,
$$

where $D^{f}$ is the Dirac staggered operator. We divided each magnetic quantum into a grid of 16 equally spaced points where we computed the operator Eq. 2.2, then we obtained the energy differences integrating over the spline intepolation of the obtained values.

The free energy suffer from $\mathscr{O}\left(b^{2}\right)$ additive ultraviolet divergencies (for a detailed discussion see [13]), which can be removed using the following prescription [10, 11]:

$$
\Delta f^{r}(b, T)=\Delta f(b, T)-\Delta f(b, 0)
$$

where the two energy differences must be computed at the same lattice spacing.

\section{Numerical results}

\subsection{Magnetic susceptibility}

In [11] we performed lattice QCD simulations using $N_{f}=2+1$ quark flavors for three different lattice spacings $\mathrm{a}=0.2173,0.1535,0.1249$. We used a Symanzik tree level improved gauge action and stout improved staggered fermions, and we chose the simulation parameters corresponding to a line of constant physics with physical value of the pion mass [11]. For all the explored lattice spacings, the spatial lattice size has been choosen so as to keep a physical extent $a L_{s} \simeq 5 \mathrm{fm}$, while we used $N_{t}=4,6,8,10,12$ temporal sizes for the finite temperature simulations. The $T=0$ simulations needed for the subtraction in Eq.(2.3) has been done using hypercubic lattices.

To measure the magnetic susceptibility of the QCD medium, we considered the free energy density expansion:

$$
f(B, T)=f(0, T)+\frac{1}{2} c_{2}(T) B^{2}+\mathscr{O}\left(B^{4}\right)
$$

From Eq 2.1 we obtain:

$$
\Delta f(b, T)=f(b, T)-f(b-1, T)=\int_{b-1}^{b} \frac{\partial f(\bar{b}, T)}{\partial \bar{b}} d \bar{b}=\frac{1}{2} c_{2}(T)(2 b-1)+\mathscr{O}\left(b^{4}\right)
$$

Then we extracted the value of the $c_{2}(T)$ coefficient fitting the free energy differences $\Delta f(b, T)$ using Eq. 3.2. The slope difference $c_{2 R}(T)=c_{2}(T)-c_{2}(T=0)$ yields to the renormalized magnetic susceptibility:

$$
\begin{array}{r}
\tilde{\chi}(T)=-\frac{|e|^{2} \mu_{0} c}{18 \hbar \pi^{2}} L_{s}^{4} c_{2 R}(T) \quad \text { SI units } \\
\hat{\chi}(T)=-\left(\frac{L^{2}}{6 \pi^{2}}\right)^{2} c_{2 R}(T) \quad \text { natural units }
\end{array}
$$



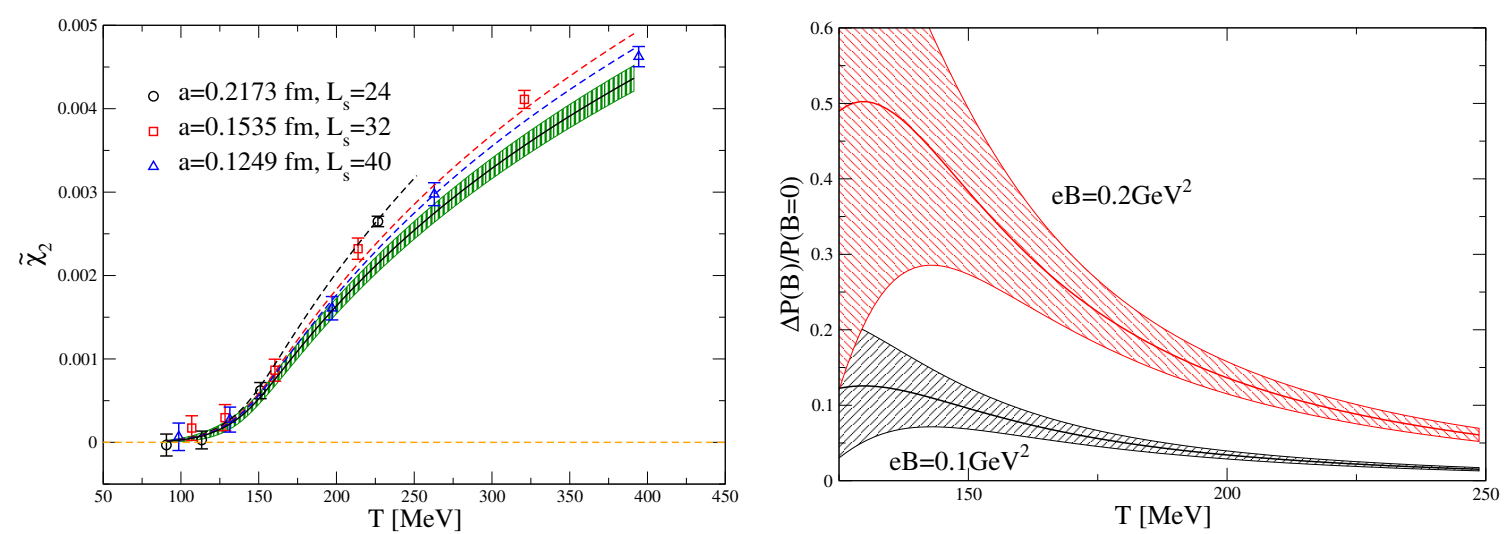

Figure 1: Left: Continuum extrapolation of the magnetic susceptibility $\tilde{\chi}(T)$ from our data at three different lattice spacings. Right: Magnetic contribution to the pressure, evaluated at two different values of the magnetic field $\mathrm{eB}=0.1 \mathrm{GeV}^{2}$ and $\mathrm{eB}=0.2 \mathrm{GeV}^{2}$. The $P(B=0)$ data have been taken from [16].

In Fig. 1 (left) we report our results for the magnetic susceptibility for the three different values of the lattice spacing. We found that strongly interacting matter is paramagnetic in all the explored range of temperatures $(100-400) \mathrm{MeV}$, with a magnetic susceptibility which sharply increases at the crossover transition, $T_{c}=(150-160) \mathrm{MeV}$.

In the low temperature region, $T<170 \mathrm{MeV}$, our data can be fitted using the ansatz $\tilde{\chi}(T)=$ $A \exp (-M / T)$, giving $M=870 \pm 260 \mathrm{MeV}$, which is in the mass range of the lightest hadrons carrying non trivial magnetic moments, like the $\rho$ meson. This behavior seems in line with an Hadron Resonance Gas (HRG) model expectation, however a direct comparison with the HRG predictions shows substantial discrepancies with our data, as we will discuss later.

In the high temperature limit, the behavior of our data is correctly described by the functional behavior $A^{\prime} \log \left(T / M^{\prime}\right)$, which is expected from the lowest order perturbative prediction at asymptotically high temperatures [15].

To perform a continuum extrapolation of our results we had to fix a definite ansatz for the $\tilde{\chi}(T)$ behavior in the continuum, since given the present number of points we cannot perform a parametrization independent continuum extrapolation. We fitted our data choosing a function which reproduces the two behaviors in the different range of temperatures:

$$
\tilde{\chi}(T)= \begin{cases}A \exp (-M / T) & T \leq \tilde{T} \\ A^{\prime} \log \left(T / M^{\prime}\right) & T>\tilde{T}\end{cases}
$$

where to obtain a continuos and differentiable matching at $T=\tilde{T}$ we impose the constraints:

$$
A^{\prime}=A M \exp (-M / \tilde{T}) / \tilde{T}, \quad M^{\prime}=\tilde{T} \exp (-\tilde{T} / M)
$$

Since our data are not precise and dense enough to extract the lattice dependence of all the parameters, we introduced the lattice spacing dependence only for a subset of fit parameters. We verified that the two subsets:

- $\left\{\tilde{T}, A, M_{0}, M_{2}\right\}$ with $M=M_{0}+a^{2} M_{2}$

- $\left\{\tilde{T}, A_{0}, A_{2}, M\right\}$ with $A=A_{0}+a^{2} A_{2}$ 

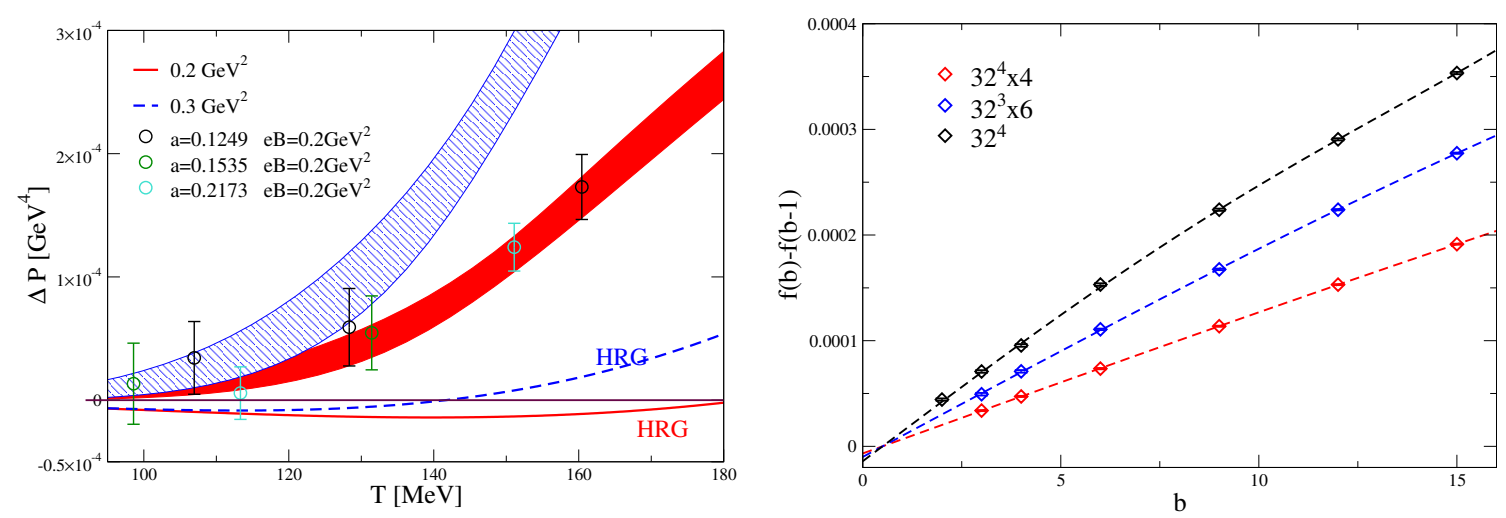

Figure 2: Left: Comparison with the HRG prediction in the low temperature phase. The two HRG lines have been computed following [17], using the same renormalization prescription of our lattice determiantion. Right: Finite free energy differences for fields up to $e B \approx 0.4 \mathrm{GeV}^{2}$. Dashed lines correspond to the fits: $f(b)-f(b-1)=\frac{1}{2} c_{2}(T)(2 b-1)+\frac{1}{4 !} c_{4}(T)\left(b^{4}-(b-1)^{4}\right)$.

return equivalent results for the continuum extrapolation. In Fig. 1 (left) we show our continuum extrapolation togheter with data points. The value of $\tilde{T}$ turns out to be compatible with the crossover temperature, $\tilde{T}=160(10) \mathrm{MeV}$.

\subsection{EoS}

Let us discuss now the effect of the magnetic fields on the equation of state of the system. Since we are dealing with a system with zero chemical potential, the pressure will be given by $P=-f$, so the change in the pressure of the system is easily obtained as $\Delta P(B)=-\Delta f_{R}=\frac{1}{2} \hat{\chi}(e B)^{2}$ and is plotted in Fig. 1 (right), as a function of $T$, for two different values of the magnetic field (we make use of our continuum extrapolation), normalized by the pressure at $e B=0$. We notice that the introduction of the magnetic field leads to a relative increase of the pressure which is larger around the crossover transition, and is in the range of $10-40 \%$ for typical field values produced during heavy ion collisions at the LHC. In the high- $T$ regime, instead, the relative increase rapidly approaches zero, as expected, since the pressure at $e B=0$ grows like $T^{4}$.

In Fig. 2 (left) we compare for a couple of values of $e B$ the pressure of the thermal medium $\Delta P(B) \simeq-\Delta f_{R}=\frac{1}{2} \tilde{\chi}(e B)^{2}$, computed from our continuum extrapolated susceptibility, with the $\mathrm{HRG}$ prediction for the same quantity (i.e. without vacuum contributions) extracted from the results reported in Ref. [17]. A few differences are clearly visible between the two: the HRG predicts a slight diamagnetic behavior for low $T$, where the pions contribution, which is indeed diamagnetic, dominates. Instead for larger fields or temperatures the main contribution to the pressure comes from the hadrons with higher spin, and the pressure behavior becomes paramagnetic. Our lattice results do not confirm this weak diamagnetic behavior for small fields and temperatures. However, for $T \lesssim 100 \mathrm{MeV}$ our current precision is not fine enough to exclude such behavior. It would be interesting in the future to further investigate this issue.

\subsection{Higher order terms}

We found that the linear approximation, Eq. 3.1, can be used for magnetic fields up to $e B=$ $0.2 \mathrm{GeV}^{2}$. To explore higher values of $e B$ we need to include higher order terms in the free energy 

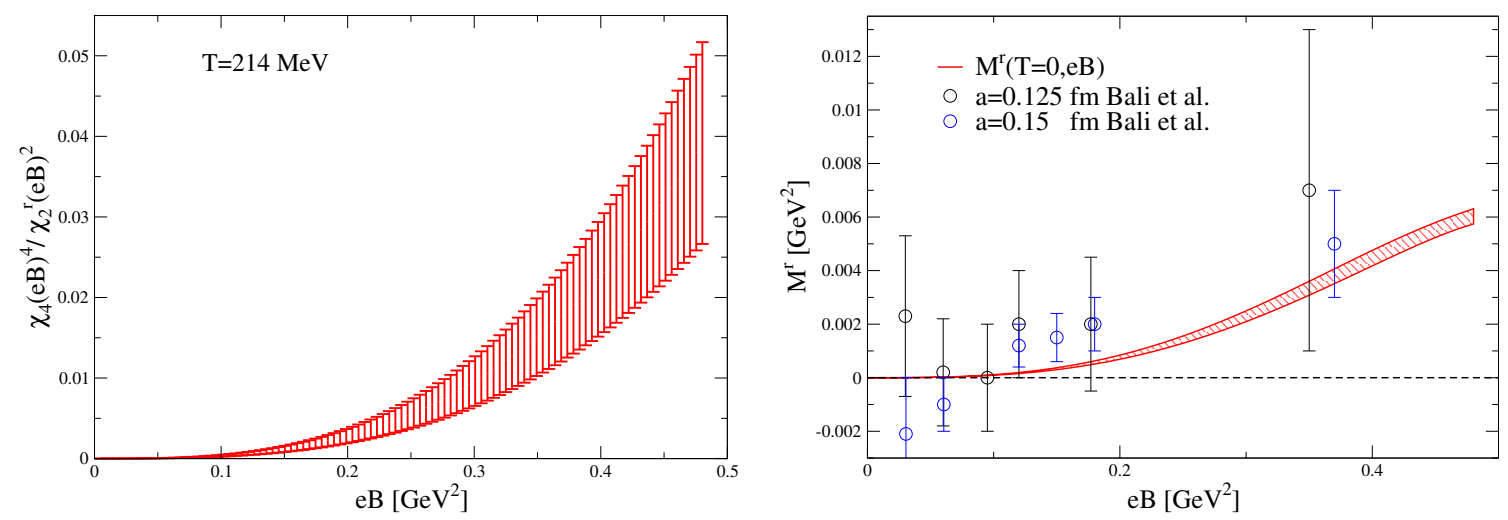

Figure 3: Left: Relative contribution of the quartic term to the first order term for $T=214 \mathrm{MeV}$, where $T=\frac{1}{a N_{t}}$. Right: Vacuum renormalized magnetization $M^{r}(0, B)$ compared with results from [12].

expantion $f(b, T)=f(0, T)+\sum_{n}\left(\frac{1}{2 n}\right) ! c_{2 n} b^{2 n}$, where the coefficients $c_{2 n}$ can be fitted from the finite energy differences and are related to the higher order dimensionless combinations:

$$
\hat{\chi}_{2 n} T^{4(n-1)}=-c_{2 n}\left(\frac{L^{2}}{6 \pi}\right)^{2 n}\left(\frac{1}{N_{t}}\right)^{4(n-1)} \quad n=1,2, \ldots \quad \text { (natural unit) }
$$

Once again we have to cure the diverging terms in the free energy density, a possible prescription is to subtract all the vacuum contributions, which means: $\hat{\chi}_{2 n}^{r}=\hat{\chi}_{2 n}(T)-\hat{\chi}_{2 n}(0)$. Using this prescription we gain information about the thermal medium alone. Alternatively, one can subtract only the $\mathscr{O}\left(b^{2}\right)$ term coming from the vacuum, which is the only diverging contribution in the free energy. We decided to use this second prescription.

We performed a preliminary computation of $\hat{\chi}_{4}$ for two temporal extents $N_{t}=4,6$, for one lattice spacing value $a=0.1535 \mathrm{fm}$. In Fig. 2 (right) we report the results of the integration Eq. 2.1 for magnetic quanta up to $b=15$. Since the single finite differences $f(b)-f(b-1)$ can be determined independently for each quantum $b$, we can easily move to large values of the magnetic field picking nonconsecutive magnetic quanta. In the fits reported in Fig. 2 (right) we used Eq. 3.7 to obtain $\hat{\chi}_{4} T^{4}=2.5(7) \cdot 10^{-4}, 2.2(2) \cdot 10^{-4}$ respectively for $N_{t}=4,6$. For the vacuum, which in our simulations refers to $N_{t}=32$, corresponding to $T \simeq 40 \mathrm{MeV}$, we found $\hat{\chi}_{4} T^{4}=1.6(2) \cdot 10^{-6}$.

An estimate of the range of fields in which the quartic term becomes relevant to the free energy determination can be easily obtained considering the ratio $\frac{\hat{\chi}_{4}(e B)^{4} / 4 !}{\hat{\chi}_{2}^{r}(e B)^{2} / 2}$. In Fig. 3 (left) we show this ratio for the case $N_{t}=6$, where we see that the quartic term $\hat{\chi}_{4}$ becomes significant for $e B \geq 0.2 \mathrm{GeV}^{2}$. We also determined $\hat{\chi}_{6}$ from out $N_{t}=32$ data, finding $\hat{\chi}_{6} T^{8}=-1.7(3) \cdot 10^{-10}$. We used these preliminary results to compute the vacuum magnetization of the system:

$$
M(e B)=e B \hat{\chi}_{2}+(e B)^{3} \frac{\hat{\chi}_{4}}{3 !}+(e B)^{5} \frac{\hat{\chi}_{6}}{5 !}+\mathscr{O}\left((e B)^{7}\right)
$$

We defined the renormalized magnetization as in [12], subtracting the $\mathscr{O}\left((e B)^{2}\right)$ term, $M^{r}=$ $M-e B \hat{\chi}_{2}$. In Fig. 3 (right) we plot our results together with the magnetization computed in [12]. We found a good agreement between the two determinations, with our determination being significantly more accurate. 


\section{Conclusions}

We presented our latest determination of the QCD magnetic properties in the presence of strong magnetic fields. We found a paramagnetic behavior of the strongly interacting matter for all the explored temperatures. Near the crossover temperature the magnetic contribution to the pressure is at the level of $10 \%$ for $e B=0.1 \mathrm{GeV}^{2}$, while for higher field, $e B=0.2 \mathrm{GeV}^{2}$, it reaches about the $40 \%$ of the total pressure. We also presented preliminary results for higher order magnetic contributions to the pressure, finding that such terms become relevant for large fields, $e B \geq 0.2 \mathrm{GeV}^{2}$.

\section{References}

[1] M. D’Elia, M. Mariti and F. Negro, Phys. Rev. Lett. 110, 082002 (2013)

[2] C. Bonati, M. D’Elia, M. Mariti, M. Mesiti, F. Negro and F. Sanfilippo, Phys. Rev. D 89, no. 11, 114502 (2014)

[3] G. Endrodi, Phys. Rev. D 90, no. 9, 094501 (2014)

[4] G. S. Bali, F. Bruckmann, G. Endrodi and A. Schafer, Phys. Rev. Lett. 112, 042301 (2014)

[5] V. Skokov, A. Y. Illarionov and V. Toneev, Int. J. Mod. Phys. A 24, 5925 (2009) V. Voronyuk, V. D. Toneev, W. Cassing, E. L. Bratkovskaya, V. P. Konchakovski and S. A. Voloshin, Phys. Rev. C 83, 054911 (2011) A. Bzdak and V. Skokov, Phys. Lett. B 710, 171 (2012)

[6] R. C. Duncan and C. Thompson, Astrophys. J. 392, L9 (1992)

[7] T. Vachaspati, Phys. Lett. B 265, 258 (1991). D. Grasso and H. R. Rubinstein, Phys. Rept. 348, 163 (2001)

[8] M. D’Elia, Lect. Notes Phys. 871, 181 (2013)

[9] G. 't Hooft, Nucl. Phys. B 153, 141 (1979), P. H. Damgaard and U. M. Heller, Nucl. Phys. B 309, 625 (1988), M. H. Al-Hashimi and U.-J. Wiese, Annals Phys. 324, 343 (2009)

[10] C. Bonati, M. D’Elia, M. Mariti, F. Negro and F. Sanfilippo, Phys. Rev. Lett. 111, 182001 (2013)

[11] C. Bonati, M. D’Elia, M. Mariti, F. Negro and F. Sanfilippo, Phys. Rev. D 89, 054506 (2014)

[12] G. S. Bali, F. Bruckmann, G. Endrodi, F. Gruber and A. Schaefer, JHEP 1304, 130 (2013)

[13] G. S. Bali, F. Bruckmann, G. Endrodi, S. D. Katz and A. Schaefer, JHEP 1408, 177 (2014)

[14] L. Levkova and C. DeTar, Phys. Rev. Lett. 112, no. 1, 012002 (2014)

[15] P. Elmfors, D. Persson and B. -S. Skagerstam, Phys. Rev. Lett. 71, 480 (1993) Astropart. Phys. 2, 299 (1994)

[16] S. Borsanyi, Z. Fodor, C. Hoelbling, S. D. Katz, S. Krieg and K. K. Szabo, Phys. Lett. B 730, 99 (2014)

[17] G. Endrodi JHEP 04, 023 (2013) 